\title{
Composición química de la fauna de acompañamiento del camarón de Veracruz (Golfo de México)
}

\author{
María Isabel Castro-González ${ }^{1}$, Jose Luis Silencio Barrita ${ }^{1}$, Maria Eugenia Juárez Silva ${ }^{1}$, \\ Sara Montaño Benavides ${ }^{1}$, Fernando Pérez-Gil Romo ${ }^{1}$ \\ 1 Subdirección de Nutrición Experimental y Ciencia de los Alimentos. Instituto Nacional de la Nutrición "Salvador \\ Zubirán”. Vasco de Quiroga 15, Thalpan 14000, México, D.F. E-mail: castro@aztlan.innsz.mx
}

Recibido 1-IX-1997. Corregido 12-II-1998. Aceptado 23-II-1998.

\begin{abstract}
Chemical composition and energy content of the shrimp by-catch used for human and livestock consumption were studied in Alvarado, Veracruz (Gulf of Mexico). Five on-board samplings were done between September 1994 and March 1995 on shrimp ships off Veracruz. The samples were frozen on board for laboratory analysis of the edible portion, they included 16 families and 22 species of fishes and 1 mollusc (squid). After moisture, the crude protein was the most abundant chemical fraction (14.3 and 19.6\%); carbohydrates were numerically variable in all groups ( 2.1 to $7.4 \%$ ); ether extract (lipids) was less abundant but variable ( 0.5 to $3.8 \%$ ), ashes were 0.10 to $0.24 \%$. Crude energy was less variable numerically ( 4.5 to $5.2 \mathrm{kcal} / \mathrm{g}$ ); 12 species are used as animal feed and only Pristipomoides aquilonaris was reported as exclusively used for this purpose: chemical composition was similar in species used for human and for livestock consumption; Anchoa hepsetus, Synodus foetens, Cynoscion arenarius, Upeneus parvus and Trichiurus lepturus are recomended for human consumption, specially for low economic level areas of Gulf of Mexico.
\end{abstract}

Key words: Chemical composition, shrimp by-catch fauna, Veracruz, Gulf of Mexico, human nutrition, animal feeding.

El término fauna de acompañamiento, sinónimo de pesca acompañante, se refiere a todas las especies de pescado, moluscos y crustáceos, incidentalmente capturados durante las operaciones de arrastre (Allsop 1983, Grande 1987). Se han realizado numerosos estudios sobre la biología y ecología de dichas especies, así como estrategias y técnicas de elaboración de productos (salchichas, paté, tortas, sopas secas, croquetas, empanizados, galletas, etc.) a partir de la fauna de acompañamiento del camarón (Arvizu 1978, Tableros y Young 1981, Pariser 1983, Allsop 1985, Corripio 1985, Ya-
ñez-Arancibia 1985, Grande 1987, Llerena y Bojorquez 1988).

Para la zona pesquera del Golfo de México se han informado proporciones FAC: camarón extremas, que van desde muy bajas (3: 1) hasta muy altas (19: 1) (Slavin 1983, Grande 1987). Estos estudios muestran una gran variabilidad tanto en la producción como en la composición, habiéndose informado alrededor de 100 especies diferentes, de las cuales entre 3 y 5 cubren generalmente el $50 \%$ de la fauna de acompañamiento y entre 7 y 10 especies, el 
75\%. En el Golfo de México se ha descrito como talla promedio del pescado de acompañamiento $17 \mathrm{~cm}$, con un peso en la mayoría de las especies capturadas menor a $0.5 \mathrm{~kg}$.

En el Puerto de Alvarado, Veracruz (Golfo de México), se ha presentado un cambio en los hábitos alimentarios de la población, con la introducción en su dieta de especies provenientes de la pesquería del camarón, sin que ésto signifique el empleo de tecnologías de producto, es decir, que estas especies se consumen en fresco. Por otro lado, se desconoce la composición química de dichas especies recolectadas en la región, ya que las Tablas sobre la composición química y el valor nutritivo de los alimentos mexicanos (Hernández et al. 1980, Mendoza et al. , Chavez et al. 1992, Chavez et al. 1996) no mencionan mas que algunas especies comestibles de productos marinos, sin informar sobre su zona de recolecta, lo cual es muy importante ya que la composición química es uno de los factores con mayor variación. Es por ello que el objetivo del presente trabajo fue identificar y cuantificar los componentes químicos proximales y la energía en diferentes especies de la fauna de acompañamiento del camarón de Alvarado, Veracruz, que son utilizadas para consumo humano y/o consumo animal a nivel regional.

\section{MATERIALES Y METODOS}

El área de estudio $\left(18^{\circ} 45^{\prime}-19^{\circ} 0^{\prime} \mathrm{N} ; 95^{\circ}\right.$ $57^{\prime}-190^{\prime} \mathrm{W}$ ) está frente a la planicie costera del área central del estado de Veracruz. Las muestras las proporcionó el Laboratorio de Ecología de la Universidad Nacional Autónoma de México. Se realizaron cinco muestreos con tres arrastres por día: muestra 1 (septiembre 1994); muestra 2 (octubre 1994); muestra 3 (noviembre 1994); muestra 4 (enero 1995); muestra 5 (marzo 1995). Los muestreos se hicieron a bordo de barcos camaroneros; del total de la captura obtenida por día se realizó un muestreo aleatorio, con lo que se obtuvieron para este estudio cinco lotes; en el Cuadro 1 se presenta el tamaño de muestra que está dado por el número de organismos que, una vez obtenida la muestra, se seleccionaron dadas sus óptimas condiciones para el análisis químico (se eliminaron del estudio organismos golpeados, fracturados o incompletos). Las muestras se mantuvieron en congelación a bordo y hasta que llegaron al laboratorio para su análisis (máximo 3 días). En el laboratorio se identificaron taxonómicamente hasta especie utilizando las claves de peces del Golfo de México de Hoose y Moore (1977) y Fisher (1978), además de una revisión complementaria de los trabajos de Castro-Aguirre (1976). De cada recolecta se separaron las especies por familia y por uso a nivel regional, constituyéndose de éste modo las siguientes categorías: I) Consumo Humano, son muestras cuyo uso se ha informado como pescado fresco, fileteado, ceviche, pescado frito o salado; y II) Consumo Animal, especies empleadas como carnada, harina de pescado o alimento para peces y aves.

Se separó la porción comestible y se sometió a los siguientes análisis: humedad por estufa de secado a $40^{\circ} \mathrm{C}$, Método 930.15 (anónimo 1990); proteína cruda: por Kjeltec, método 976.05 (anónimo 1990); cenizas: con mufla a $500^{\circ} \mathrm{C}$, método 492.05 (21); extracto etéreo: extracción con éter de petróleo, método 932.02 (anónimo 1990) e hidratos de carbono que se obtuvieron por la diferencia entre la sumatoria de las fracciones mencionadas menos 100 . La energía bruta se cuantificó con la bomba calorimétrica (Tejada 1985). Las determinaciones se realizaron por triplicado obteniéndose porcentajes de recuperación de 98.05 a $99.74 \%$. Se presenta la media y desviación estándard de los resultados de los análisis químicos realizados a las especies obtenidas en las diferentes recolectas.

\section{RESULTADOS}

De las recolectas analizadas se identificaron un total de 16 familias con 22 especies de 
peces, y una familia con una especie de moluscos, en el Cuadro 1 se presentan los datos generales de las especies analizadas; la talla está dada por la media de las diferentes recolectas. Las familias Mulleidae (Upeneus parvus) y Serranidae (Diplectrum bivittatum), se identificaron en cinco y cuatro recolectas, por lo que dichos grupos se consideraron de mayor incidencia. De mediana incidencia (tres recolectas) son la mayoría de las especies: Engraulidae (Anchoa hepsetus), Scianidae (Cynoscion arenarius), Bothidae (Syacium gunteri), Carangidae (Selene spixii, Trachiurus lathami), Clupleidae (Harengula jaguana), Synodontidae (Synodos foetens) y Trichuridae (Trichurus lepturus). De baja incidencia (una a dos recolectas) fueron las familias restantes. El molusco identificado perteneció a la familia Loligidae, específicamente el calamar (Loligo pealei), que se presentó en las recolectas 2,3 y 4 y su empleo a nivel regional es para consumo humano y carnada.

La humedad de las muestras frescas fue desde $72.5 \%$ (D. auratus) hasta $80.02 \%$ (H. jaguana) y se encuentra en el intervalo de humedad de fauna de acompañamiento del camarón señalado por Morrisey (1985) (73.76 -79.37\%) y por Corripio (1985) (77.0-79.0\%).

Consumo humano: El contenido de proteína cruda (PC) en las especies para consumo humano (Cuadros 2 y 3 ) se presentó con un intervalo entre $11.88 \%$ (H. jaguana) y $19.62 \%$ (T. lepturus). Se observa que el $67.6 \%$ de las especies tienen más de $16 \%$ de proteína cruda y un $48 \%$ entre 15 y $17 \%$ de esta fracción. Las cenizas (porción mineral) (Cuadros 2 y 3), se

\section{CUADRO 1}

Especies de la fauna de acompañamiento del camarón de Alvarado, Veracruz consumidas regionalmente (1994 -1995)

$\begin{array}{llllll}\text { Familia } & \text { Nombre científico } & \text { Uso } & \begin{array}{l}\text { Colecta } \\ \text { número }\end{array} & \begin{array}{l}\text { Tamaño } \\ \text { muestra }\end{array} & \begin{array}{c}\text { Talla } \\ \text { promedio }\end{array} \\ & & & & & \\ \text { Balistidae } & \text { Balistes capriscus } & \mathrm{CH} & 3 & 5 & 20 \pm 2.1 \\ \text { Bothidae } & \text { Cyclopsetta } \text { chittendeni } & \mathrm{CH} & 1,3 & 4,5 & 30 \pm 6.5 \\ & \text { Syacium gunteri } & \mathrm{CH} & 1,2,3 & 6,8,12 & 10 \pm 3.6 \\ \text { Branchiostegidae } & \text { Caulolatilus intermedius } & \mathrm{CH} & 1 & 3 & 14 \pm 1.7 \\ \text { Carangidae } & \text { Selene vomer } & \mathrm{CH} & 1,2,3 & 10,8,7 & 23 \pm 2.0 \\ & \text { Trachurus lathami } & \mathrm{CH} & 3,4,5 & 2,1,3 & 15 \pm 4.3 \\ \text { Clupeidae } & \text { Harengula jaguana } & \mathrm{CH} & 2,4,5 & 1,1,4 & 10 \pm 1.6 \\ \text { Elopidae } & \text { Elops saurus } & \mathrm{CH} & 3 & 2 & 61 \pm 4.6 \\ \text { Engraulidae } & \text { Anchoa hepsetus } & \mathrm{CH} & 2,3,5 & 11,6,7 & 11 \pm 2.5 \\ \text { Gerreidae } & \text { Diapterus auratus } & \mathrm{CH} / \mathrm{CA} & 1 & 6 & 10 \pm 3.7 \\ \text { Lutjanidae } & \text { Pristipomoides aquilonaris } & \mathrm{CA} & 1,3 & 3,5 & 12 \pm 2.2 \\ \text { Mulleidae } & \text { Upeneus parvus } & \mathrm{CH} / \mathrm{CA} & 1,2,3,4,5 & 2,5,3,2,4 & 15 \pm 1.3 \\ \text { Pomadasydae } & \text { Conodon nobilis } & \mathrm{CH} & 1,3 & 6,9 & 25 \pm 1.1 \\ \text { Sciaenidae } & \text { Cynoscion arenarius } & \mathrm{CH} / \mathrm{CA} & 1,2,4 & 3,2,2 & 35 \pm 3.3 \\ & \text { Cynoscion } \text { nothus } & \mathrm{CH} / \mathrm{CA} & 2,3 & 4,2 & 26 \pm 2.3 \\ & \text { Micropogonias furnieri } & \mathrm{CH} / \mathrm{CA} & 2,3 & 2,1 & 30 \pm 5.4 \\ \text { Serranidae } & \text { Diplectrum bivittatum } & \mathrm{CH} & 1,3,4,5 & 3,6,4,9 & 11 \pm 2.9 \\ & \text { Diplectrum formosum } & \mathrm{CH} & 3 & 2 & 13.5 \pm 4 \\ \text { Synodontidae } & \text { Synodus foetens } & \mathrm{CH} / \mathrm{CA} & 1,2,3 & 3,11,5 & 25 \pm 3.2 \\ \text { Trichuridae } & \text { Trichiurus lepturus } & \mathrm{CH} / \mathrm{CA} & 1,2,3 & 2,2,3 & 62 \pm 8.3 \\ \text { Triglidae } & \text { Prionotus roseus } & \mathrm{CH} / \mathrm{CA} & 4,5 & 1,3 & 12 \pm 1.8 \\ & \text { Prionotus rubio } & \mathrm{CH} / \mathrm{CA} & 4,5 & 4,4 & 10 \pm 3.1 \\ \text { Loligidae } & \text { Loligo pealei } & \mathrm{CH} / \mathrm{CA} & 2,3,4 & 2,4,2 & 26 \pm 3.2\end{array}$

$\mathrm{CH}=$ consumo humano, $\mathrm{CA}=$ consumo animal, 1 = número de organismos analizados por recolecta, respectivamente 
presentaron en un intervalo entre $0.11 \%$ ( $T$. lepturus) y $0.24 \%$ (C. nobilis); el contenido de cenizas en los moluscos es menor a $0.10 \%$.

Es evidente que el contenido de extracto etéreo en las diferentes especies de fauna de acompañamiento del camarón, es la fracción más variable, dado que los valores obtenidos oscilaron entre $0.5 \%$ ( $S$. foetens, $P$. roseus y $P$. rubio) y $3.8 \%$ (E. saurus); sin embargo, el valor máximo observado se presenta en la especie $U$. parvus, con un contenido de extracto etéreo de $4.2 \%$. La mayoría de las especies presentaron valores menores a $3 \%$.

Más abundantes que la fracción lipídica fueron sin duda los hidratos de carbono. Como se observa en los Cuadros 2 y 3 los valores van desde $2.1 \%$ (C. arenarius) hasta $7.4 \%$ (C. nobilis). La energía, producto terminal de los nutrimentos energéticos, presentó valores con la menor variación numérica, entre las diferentes especies. En los Cuadros 2 y 3 se presenta el contenido de energía bruta con valores entre 4.2 $\mathrm{kcal} / \mathrm{g}$ (C. nobilis) y $5.7 \mathrm{kcal} / \mathrm{g}$ (U. parvus).Otro grupo detectado con frecuencia en la fauna de acompañamiento del camarón fue el de los moluscos; la fracción mas abundante en el calamar (L. pealei) fue la proteína cruda (Cuadro 2) con valores de $17.4 \%$, considerándose esta especie como una buena fuente proteica. El contenido de cenizas fue de $0.07 \%$ y el extracto etéreo de $0.7 \%$; los hidratos de carbono se cuantificaron en $1.6 \%$. La energía bruta fue de (4.8 kcal/g).

Consumo animal: Se identificaron 12 especies de fauna de acompañamiento del camarón que son utilizadas a nivel regional como alimento para animales y carnada (Cuadro 1). La especie $P$. aquilonaris se identificó como de uso exclusivo en alimentación animal. La proteína cruda (Cuadro 2) se presentó en un intervalo de $14.3 \%$ (P. rubio) a $19.6 \%$ (T. lepturus). Al igual que con las muestras para consumo humano, la fracción mas variable numéricamente fue el extracto etéreo, con valores muy bajos en especies como $P$. rubio, $P$. roseus y $S$. foetens $(0.5 \%)$; valores intermedios en la mayoría de las especies, entre $0.7 \%$ (T. leptu-

\section{CUADRO 2}

Composición química de las especies de acompañamiento del camarón de Alvarado, Veracruz (1994 -1995), empleadas para consumo humano y/o animal

$\begin{array}{lrlllll}\text { Especie } & \text { Humedad \% } & \text { Cenizas \% } & \text { P.C. } \% & \text { E.E. \% } & \text { H.C. \% } & \text { Energía kcal } / g \\ \text { Mayor } & \text { incidencia } & & & & & \\ \text { U. parvus } & 74.5 \pm 0.02 & 0.15 \pm 0.01 & 16.45 \pm .14 & 4.2 \pm 0.04 & 4.72 & 5.7 \pm .09 \\ \text { D. bivittatum } & 73.9 \pm 0.03 & 0.19 \pm 0.02 & 17.77 \pm .06 & 2.1 \pm 0.001 & 6.15 & 5.5 \pm .007\end{array}$

$\begin{array}{lllllll}\text { Mediana } & \text { incidencia } & & & & \\ \text { S. gunteri } & 77.2 \pm 0.03 & 0.18 \pm 0.07 & 16.84 \pm .07 & 0.9 \pm 0.03 & 4.84 & 4.8 \pm .02 \\ \text { C. } \text { arenarius } & 76.2 \pm 0.05 & 0.17 \pm 0.01 & 18.34 \pm .04 & 3.2 \pm 0.01 & 2.1 & 4.8 \pm .10 \\ \text { A. hepsetus } & 77.6 \pm 0.004 & 0.13 \pm 0.1 & 15.74 \pm .01 & 2.0 \pm 0.001 & 4.51 & 5.3 \pm .05 \\ \text { H. jaguana } & 75.4 \pm 0.01 & 0.20 \pm 0.003 & 15.72 \pm .02 & 2.1 \pm 0.001 & 6.56 & 5.2 \pm .09 \\ \text { T. lepturus } & 76.32 \pm 0.06 & 0.11 \pm 0.02 & 19.62 \pm .03 & 0.7 \pm 0.02 & 3.27 & 4.8 \pm .10 \\ \text { S. foetens } & 78.30 \pm 0.01 & 0.14 \pm 0.02 & 16.93 \pm .09 & 0.8 \pm 0.06 & 3.79 & 4.4 \pm .08 \\ \text { S. vomer } & 75.4 \pm 0.06 & 0.15 \pm 0.01 & 17.67 \pm 0.6 & 2.3 \pm 0.06 & 4.44 & 4.9 \pm .04 \\ \text { T. lathami } & 76.5 \pm 0.03 & 0.16 \pm 0.01 & 17.54 \pm .01 & 0.9 \pm 0.09 & 4.85 & 5.5 \pm .13 \\ \text { L. pealei } & 80.21 \pm 0.04 & 0.07 \pm 0.01 & 17.44 \pm .02 & 0.7 \pm 0.02 & 1.60 & 4.9 \pm .06\end{array}$

P.C. = proteína cruda, E.E. $=$ extracto etéreo, H. C. $=$ hidratos de carbono. 


\section{CUADRO 3}

Composición química de las especies de acompañamiento del camarón de Alvarado, Veracruz (1994 -95), empleadas para consumo humano y/o animal, de baja incidencia

$\begin{array}{lcccccc}\text { Especie } & \text { Humedad \% } & \text { Cenizas \% } & \text { P.C. \% } & \text { E.E. \% } & \text { H.C. \% } & \text { Energía kcal/g } \\ & & & & & & \\ \text { C.intermedius } & 77.8 \pm .05 & 0.16 \pm 0.09 & 16.0 \pm 0.11 & 2.1 \pm 0.09 & 4.95 & 5.09 \pm .04 \\ \text { M.furnieri } & 73.4 \pm 0.04 & 0.19 \pm 0.02 & 17.98 \pm .07 & 2.6 \pm 0.03 & 5.85 & 5.02 \pm .03 \\ \text { D.auratus } & 72.5 \pm 0.09 & 0.18 \pm .004 & 17.92 \pm .04 & 2.8 \pm 0.03 & 6.58 & 5.21 \pm .03 \\ \text { C.nobilis } & 73.8 \pm 0.01 & 0.24 \pm 0.02 & 17.27 \pm .06 & 1.3 \pm 0.11 & 7.36 & 4.72 \pm .02 \\ \text { C.nothus } & 75.45 \pm .01 & 0.14 \pm 0.01 & 18.69 \pm .04 & 1.3 \pm 0.002 & 4.41 & 5.1 \pm 0.03 \\ \text { C.chittendeni } & 74.65 \pm .01 & 0.18 \pm 0.03 & 18.63 \pm .07 & 0.9 \pm 0.03 & 5.60 & 5.3 \pm .08 \\ \text { P.roseus } & 78.3 \pm .003 & 0.23 \pm .01 & 14.68 \pm .01 & 0.5 \pm 0.06 & 6.25 & 4.54 \pm .02 \\ \text { B.capriscus } & 77.9 \pm .01 & 0.14 \pm .02 & 17.10 \pm .05 & 1.0 \pm 0.03 & 3.86 & 4.63 \pm .13 \\ \text { D.formosum } & 76.35 \pm .02 & 0.19 \pm .02 & 15.57 \pm .01 & 2.13 \pm 0.18 & 5.75 & 4.91 \pm .01 \\ \text { E.saurus } & 74.2 \pm .002 & 0.13 \pm 0.01 & 17.10 \pm .17 & 3.8 \pm 0.03 & 4.75 & 5.21 \pm .04 \\ \text { P.rubio } & 79.2 \pm 0.09 & 0.21 \pm .001 & 14.30 \pm .01 & 0.5 \pm 0.05 & 5.83 & 4.51 \pm .01 \\ \text { P. aquilonaris } & 72.7 \pm 0.41 & 0.63 \pm 0.02 & 17.74 \pm .33 & 2.02 \pm 0.06 & 6.91 & 5.46 \pm .08\end{array}$

P.C. $=$ proteína cruda, E.E. $=$ extracto etéreo, H.C. $=$ hidratos de carbono

rus) y $2.1 \%$ (D. bivittatum), y sólo $U$. parvus con un valor muy alto (4.2\%). El contenido de cenizas fue bajo en $U$. parvus $(0.15 \%)$, en $S$. foetens y $C$. nobilis $(0.24 \%)$. Los hidratos de carbono fueron muy variables, los valores van desde $2.1 \%$ (C. arenarius) hasta $7.4 \%$ (C. nobilis), la mayoría de las especies tuvieron valores alrededor del $4 \%$. La energía bruta se presenta con poca variación, entre $4.2 \mathrm{kcal} / \mathrm{g}(C$. nobilis) y $5.7 \mathrm{kcal} / \mathrm{g}$ en $U$. parvus; la mayoría de las especies tuvieron un contenido de alrededor de $4.5 \mathrm{kcal} / \mathrm{g}$.

Familias con mayor incidencia y con incidencia intermedia: La composición proximal de las especies con mayor incidencia (Cuadro 2) en las capturas de camarón es poco variable entre sí y dados sus valores de proteína cruda y de extracto etéreo, principalmente, se podrían considerar como potenciales de explotación y aprovechamiento para consumo humano y animal. En las especies con incidencia intermedia; los componentes químicos presentan una mayor variación numérica entre sí, sobre todo para hidratos de carbono. Su aprovechamiento en la alimentación humana y animal debe ser con- siderado dado su contenido, a modo general, de proteína cruda y extracto etéreo.

\section{DISCUSION}

La gran diversidad de especies procedentes de la fauna de acompañamiento del camarón del Puerto de Alvarado, Veracruz, México constituyen un rico potencial para la alimentación humana y animal, aunado esto a su composición química, principalmente por su contenido de proteína y lípidos. Las especies analizadas son consumidas actualmente en fresco por la población del Puerto de Alvarado, Veracruz, donde aún no se ha introducido ningún tipo de tecnología para su aprovechamiento; por esto mismo, los criterios de selección utilizados por estos consumidores se basan en primer lugar en el aspecto, seguido de la talla y de las propiedades organolépticas de la especie. En el Cuadro 1 se observa un intervalo en la talla promedio entre $10.0 \mathrm{~cm}(S$. gunteri) y $60.0 \mathrm{~cm}$ (E. saurus y $T$. lepturus). Grande (1987) mencionó para especies, de la fauna de acompañamiento, del Golfo de México una 
talla promedio de $17.0 \mathrm{~cm}$; sin embargo, en el presente trabajo se observa que la talla promedio de las especies utilizadas para consumo humano fue mayor. De las especies analizadas, doce de ellas se utilizan exclusivamente para consumo humano; diez especies se utilizan tanto para consumo humano como animal y sólo una especie ( $P$. aquilonaris) es exclusiva para consumo animal.

En los peces la proteína juega un papel muy importante para el mantenimiento, regeneración de los tejidos y crecimiento o formación de nuevas estructuras proteicas (Higuera 1989); la proteína cruda (Cuadros 2 y 3) fue la fracción química más abundante, después de la humedad, en todas las especies, independientemente de la familia, época de recolecta e incidencia; la proteína cruda en las especies de mayor incidencia (Cuadro 2) tuvo un intervalo de 16 a $17 \%$ y de 15 a $19 \%$ de proteína cruda se presentó en las especies de incidencia intermedia; entre un 14 y $18 \%$ de proteína cruda se obtuvo en los organismos de menor incidencia (Cuadro 3). De acuerdo a Van de Kamp y Wykes (1992) la porción de músculo comestible en el pescado es generalmente de $18 \%$ de proteína con un contenido entre 1 y $2 \%$ de cenizas, cuando se reporta en base húmeda. Los datos sobre la composición de pescado entero indican mayor contenido de cenizas y un poco menos de proteína. Sin embargo, desde el punto de vista de la nutrición el contenido de proteína cruda de todas las especies es tan bueno como lo informado para especies comerciales de consumo cotidiano en México, cuyos valores están entre 16.70 (todas las variedades de lenguado) y $20.10 \%$ (cabrilla) (Chávez et al. 1992, 1996). Estos valores concuerdan con lo mencionado por Poulter (Morrissey 1985) quien concluye que la composición proximal de la mayoría de los peces capturados como fauna de acompañamiento del camarón es similar entre sí, a excepción de la proteína cru$\mathrm{da}$, con un intervalo ente 15.2 y $25.4 \%$ y la grasa entre 1.31 y $6.13 \%$.Por otro lado, son bien conocidos los efectos benéficos de los productos marinos en la salud y alimentación, principalmente por su contenido de ácidos grasos (que forman parte del extracto etéreo) (Nelson 1991, Uauy y Valenzuela 1992, Van de Kamp y Wykes 199). Las especies $U$. parvus (mayor incidencia), C. arenarius (mediana incidencia) y $E$. saurus (baja incidencia) presentaron los valores más altos de extracto etéreo, por lo que en estas especies será muy conveniente un análisis más específico sobre la concentración de ácidos grasos. Los glúcidos constituyen el grupo de nutrimentos más controvertido en la alimentación de los peces, al parecer sus requerimientos son nulos; mencionádose la utilización de glúcidos absorbibles con fines de ahorro proteico, favoreciendo el crecimiento y la retención de nitrógeno ( $\mathrm{Za}-$ mora y Echeverria 1989). A pesar de que el contenido de hidratos de carbono fue mayor, en todas las especies, que los lípidos (extracto etéreo), desde un punto de vista de la nutrición éstos últimos aportan elementos de mayor relevancia en el proceso salud-enfermedad, como son los ácidos grasos $\omega-3$. En las especies carnívoras (como es el caso de las aquí mencionadas), que tienen una capacidad limitada para utilizar hidratos de carbono de alto peso molecular como fuente de energía, los lípidos juegan un papel importante al respecto, metabolizandose antes que las proteinas en los peces (Watanabe 1989). Según Van de Kamp y Wykes (1991) el músculo negro de algunos peces puede contener una mayor cantidad de triglicéridos que el músculo blanco y la deposición de éstos en el músculo comestible está sujeto a variaciones estacionales en todas las especies marinas. Para el hombre, las principales fuentes de hidratos de carbono están dadas por los cereales y tubérculos. De la misma forma, el contenido mineral, representado por las cenizas, es bajo en los organismos analizados y no representa una buena fuente.

La energía en los peces está dada por las proteínas y los lípidos, principalmente, los valores de energía bruta de los hidratos de carbono y las proteínas son similares (4.12 y 5.67 $\mathrm{kcal} / \mathrm{g}$, respectivamente) pero el de las grasas es más. alto $(9.45 \mathrm{kcal} / \mathrm{g})$, por lo que la varia- 
ción en el contenido lipídico de un organismo tendrá gran influencia sobre el contenido de energía bruta; la concentración de minerales (cenizas) no influye en el valor de energía bruta, ya que los componentes inorgánicos no son combustibles (Young 1989). La energía bruta fue mayor en las especies de mediana incidencia, comparada con las otras especies.

En cuanto al único molusco de la fauna de acompañamiento del camarónque se aprovecha ( $L$. pealei), este resultó ser buena fuente de proteína cruda pero muy pobre en lípidos.

Especies como A. hepsetus, S. foetens, $C$. arenarius, $U$. parvus y $T$. lepturus, que son regionalmente ya aprovechadas en alimentación humana, deben ser consideradas para estudios multidisciplinarios mas detallados dada su constante presencia en la captura de camarón, su contenido de proteína cruda y extracto etéreo, así como la elevada concentración de energía bruta. Por lo que así mismo se recomienda un uso mas extenso de estas especies. Por otro lado, la composición química de las especies para consumo humano y animal fue similar y nutricionalmente tienen el mismo valor; por lo tanto $U$. parvus, $C$. arenarius, $T$. lepturus y $L$. pealei, por su frecuencia en las capturas, su valor nutricional y el uso, regionalmente establecido, se recomiendan como alimento para animales. Con la información presentada se pretende contribuir de algún modo, para una mayor explotación y aprovechamiento de las especies pertenecientes a la fauna de acompañamiento del camarón de Alvarado, Veracruz, así como aportar nueva información sobre el contenido de algunos nutrimentos, en recursos marinos consumidos frecuentemente.

\section{AGRADECIMIENTOS}

Los autores expresan su agradecimiento a Jonatan Franco y Edgar Peláez del Lab. de Ecología, UNAM-Iztacala, por el aporte de muestras biológicas y su identificación taxonómica.

\section{RESUMEN}

La composición química aproximada y la energía de las especies acompañantes del camarón utilizadas para consumo humano y animal se estudió en Alvarado, Vereacruz (Golfo de México). Cinco muestreos se realizaron entre septiembre de 1994 y marzo de 1995 en barcos camaroneros. Las muestras se congelaron a bordo para los análisis de laboratorio de la porción comestible. Los resultados indicaron 16 familias, 22 especies de peces y 1 molusco (calamar). Después de la humedad, la fracción química más abundante fue la proteína cruda con valores entre 14.3 y $19.6 \%$. Los hidratos de carbono fueron desde 2.1 a $7.4 \%$. El extracto etéreo (lípidos) fue menos abundante pero variable numéricamente $(0.5$ a $3.8 \%)$, las cenizas se cuantificaron entre 0.10 y $0.24 \%$. La energía presentó la menor variación numérica $(4.5$ a $5.2 \mathrm{kcal} / \mathrm{g}) .12$ especies son empleadas como alimento animal y solo Pristipomoides aquilonaris se reportó como de uso exclusivo para este propósito; la composición química fue similar a la de las especies de consumo humano. Anchoa hepsetus, Synodus foetens, Cynoscion arenarius, Upeneus parvus y Trichiurus lepturus, se recomiendan para alimentación humana, especialmento por su bajo costo en la costa del Golfo de México.

\section{REFERENCIAS}

Allsop, H. 1983. Introduction, p.7-20. In FAO/CIID (ed.). Fish by-catch...Bonus from the sea. International Development Research Center. Vancouver, Canadá.

Allsop, H. 1985. La fauna acompañante del camarón. Perspectivas y Manejo, p.635-644. In A. Yañez-Arancibia (ed.). Recursos Pesqueros Potenciales de México: La Pesca Acompañante del Camarón. UNAM. México, D.F.

Anónimo. 1990. Official Methods of Analysis of the Association of Official Analytical Chemists., Washington, D.C. $684 \mathrm{p}$.

Arvizu, J. 1978. Aprovechamiento de la Fauna de Acompañamiento del Camarón. La Alternativa Tecnológica. Universidad Autónoma de Baja California Sur, La Paz, B.C.S. 109 p.

Castro-Aguirre, J.L. 1976. Catálogo Sistemático de los Peces Marinos que penetran a las Aguas Continentales de México con Aspectos Zoogeográficos y Ecológicos (Claves). Instituto Nacional de Pesca, México, D.F. 298 p.

Corripio, E. 1985. Fauna de Acompañamiento del Camarón y su Aprovechamiento en la Plataforma 
Continental de Tamaulipas, Golfo de México, p. 677692. In A. Yañez-Arancibia (ed.) Recursos Pesqueros Potenciales de México: La Pesca Acompañante del Camarón. UNAM. México, D.F.

Chávez, M., M. Hernández \& J. Roldán. 1992. Tablas de uso práctico del valor nutritivo de los alimentos de mayor consumo en México. C.N.A./INNSZ, México, D.F. 34 p.

Chávez, M., A. Chávez, F. Pérez-Gil, A. Roldán, A. Ledesma, L. Hernández, E. Mendoza \& A. Chaparro. 1996. Tablas del Valor Nutritivo de los Alimentos de Mayor Consumo en México. INNSZ/PAX/INC, México, D.F. $330 \mathrm{p}$.

Fisher, W. 1978. Species Identification Sheets for Fishery and Purposes. Western Central Atlantic. FAO, Roma. 236p.

Grande, V.J.M. 1987. Estrategias de acción para el aprovechamiento de la fauna de acompañamiento del camarón en México. Sria. de Pesca. I.N.P., México, D.F. 32 p.

Hernández, M., A. Chávez \& H. Bourges. 1980. Valor Nutritivo de los Alimentos, Tablas de Uso Práctico. INNSZ, México, D.F. 34 p.

Higuera, M. 1989. Requerimientos de proteína y aminoácidos en peces, p.53-98. In FAO (ed.). Nutrición y Alimentación de peces y camarones cultivados. ONU/FAO, Roma.

Hoose, H.D. \& Moore, R.H. 1977. Fishes of the Gulf of México. Texas, Louisiana and Adjacent Waters (Claves). Texas A\&M University, Texas. 309 p.

Llerena, J. \& Bojorquez, L. 1988. Aprovechamiento de la Fauna de Acompañamiento del Camarón. Simposium de Investigación sobre Alimentos y Nutrición, Tampico, México. 24p.

Mendoza, E., H. Bourges, L. Morales \& A.Chávez. 1987. Tablas de Composición de Alimentos Industrializados. INNSZ, México, D.F. 34 p.

Morrisey, M. 1985. El Uso de la Fauna de Acompañamiento del Camarón para Alimentos Humanos, p. 645-676. In A. Yañez-Arancibia (ed.). Recursos Pesqueros Potenciales de México: La Pesca Acompañante del Camarón. UNAM. México.
Nelson, G. J. 1991. Health effects of dietary fatty acids. American Oil Chemists Society, Champaign, Illinois. 288p.

Pariser, E. 1983. By-Catch for Human Consumption, p.3742. In FAO/CIID (ed.). Fish by-catch...Bonus from the sea. International Development Research Center. Vancouver, Canadá.

Slavin, J. 1983. Utilization of the Shrimp By-Catch, p. 2128. In FAO/CIID (ed.). Fish by catch...Bonus from the sea. International Development Research Center. Vancouver, Canadá.

Tableros, M. \& R. Young. 1981. Behaviour of the mechanically separated flesh of some cornmon fish species of the Mexican shrimp by-catch during storage at-20 C. J. Food. Technol. 16: 199-212.

Tejada, I. 1985. Manual de Laboratorio para Análisis de Ingredientes Utilizados en la Alimentación Animal. PAIPEME, México. 387 p.

Uauy, D. R. \& A. Valenzuela. 1992. Marine oils as a source of omega-3 fatty acids in the diet: How optimize the health benefits?. Prog. Food Nutr. Sci. 16: 199-243.

Van de Kamp, J. \& A. Wykes. 1992. Effects of fish oils and polyunsaturated omega 3 fatty acids in healt and disease. National Institute of Health, Bethesda, Maryland. 36p.

Watanabe, T. 1989. Requerimientos de ácidos grasos y nutrición lipídica en los peces, p. 99-166. In FAO (ed.). Nutrición y Alimentación de peces y camarones cultivados. Manual de capacitación. ONU, Roma.

Young, Ch. 1989. La energía en la nutrición de los peces, p.197-244. In FAO (ed.). Nutrición y Alimentación de peces y camarones cultivados. Manual de capacitación. ONU, Roma.

Yañez-Arancibia, A. 1985. Recursos Pesqueros Potenciales de México: La Pesca Acompañante del Camarón. UNAM, México, D.F. 748p.

Zamora, S. \& G. Echevarria. 1989. Los hidratos de carbono en la nutrición de los peces, p.167-196. In FAO (ed.). Nutrición y Alimentación de peces y camarones cultivados. Manual de capacitación. ONU, Roma. 